\title{
Reply to Comment on \\ "Hierarchical Bayesian space-time interpolation versus spatio-temporal BME approach” by Kolovos (2009)
}

\author{
I. Hussain, J. Pilz, and G. Spoeck \\ Department of Statistics, University of Klagenfurt, Klagenfurt, Austria
}

As correctly pointed out by A. Kolovos, in our paper (Hussain et al., 2010) we applied BME kriging and compared it to hierarchical Bayesian interpolation (HBI) in the context of spatiotemporal prediction of precipitation in Pakistan during Monsoon periods of 1974-2000. We could not make use of the distinctive features of BME such as soft data and physical knowledge, since these were simply not available in our context. It is in this respect that we agree with A. Kolovos' statement that our comparison with BME is limited. By no means we intended a misrepresentation of BME methodology and/or BME software, respectively. BME really provides a novel and very flexible approach to spatiotemporal data analysis, cf. Christakos (2000). Due to its flexibility the BME methodology is, however, not easy to apply in routine analyses. The specification of uncertainty aspects, soft data constraints and physical knowledge requires a high level of expertise, not only regarding the context, but also regarding subjective probability modeling. We therefore welcome A. Kolovos' suggestion to provide follow-up similar studies to foster further comparisons between BME and other geostatistical techniques, including ours.

\section{References}

Christakos, G.: Modern Spatiotemporal Geostatistics, Oxford Univ. Press, New York, 2000.

Hussain, I., Pilz, J., and Spoeck, G.: Hierarchical Bayesian spacetime interpolation versus spatio-temporal BME approach, Adv. Geosci., 25, 97-102, doi:10.5194/adgeo-25-97-2010, 2010. 